\title{
Determination of structural chirality of berlinite and quartz using resonant $x$-ray diffraction with circularly polarized $x$-rays
}

\author{
Yoshikazu Tanaka, ${ }^{1}$ Taro Kojima, ${ }^{1,2}$ Yasutaka Takata, ${ }^{1,2}$ Ashish Chainani, ${ }^{1}$ Stephen W. Lovesey, ${ }^{3}$ Kevin S. Knight, ${ }^{4}$ \\ Tomoyuki Takeuchi, ${ }^{1,5}$ Masaki Oura, ${ }^{1}$ Yasunori Senba, ${ }^{5}$ Haruhiko Ohashi, ${ }^{5}$ and Shik Shin ${ }^{1}$ \\ ${ }^{1}$ RIKEN SPring-8 Center, Sayo, Hyogo 679-5148, Japan \\ ${ }^{2}$ Department of Complexity Science and Engineering, The University of Tokyo, Kashiwa, Chiba 277-8561, Japan \\ ${ }^{3}$ ISIS Facility and Diamond Light Source, Rutherford Appleton Laboratory, Oxfordshire OX110QX, United Kingdom \\ ${ }^{4}$ ISIS Facility, Rutherford Appleton Laboratory, Oxfordshire OX110QX, United Kingdom \\ 5 Japan Synchrotron Radiation Research Institute (JASRI), Sayo, Hyogo 679-5198, Japan
}

(Received 17 December 2009; revised manuscript received 9 March 2010; published 12 April 2010)

\begin{abstract}
Many proteins, sugars, and pharmaceuticals crystallize into two forms that are mirror images of each other (enantiomers) such as our right and left hands (chiral). Berlinite $\left(\mathrm{AlPO}_{4}\right)$ and low quartz $\left(\mathrm{SiO}_{2}\right)$ have enantiomers belonging to a space-group pair, $P 3_{1} 21$ (right-handed screw) and $P 3_{2} 21$ (left-handed screw). We use circularly polarized resonant $\mathrm{x}$-ray diffraction to study structural chirality. Our results demonstrate that positive and negative circularly polarized X-rays at the resonant energy of berlinite (Al $1 s$ edge) and low quartz ( $\mathrm{Si} 1 s$ edge) can distinguish the absolute structure (right or left-handed screw) of an enantiomer. The advantage of our method is that the measurement of only one space-group forbidden reflection is enough to determine the chirality. This method is applicable to chiral motifs that occur in biomolecules, liquid crystals, ferroelectrics and antiferroelectrics, multiferroics, etc.
\end{abstract}

DOI: 10.1103/PhysRevB.81.144104

PACS number(s): 61.05.cp, 42.70.Ce

\section{INTRODUCTION}

It is well known that low quartz (or $\alpha$ quartz) exists in nature in two crystal structure forms which are exact mirror images of each other. The two structures belong to two different space groups. One simple way to distinguish them is by passing visible light through them and measuring the plane of polarization of the transmitted light. The polarization plane of light gets rotated in a clockwise or counterclockwise direction, and is used to distinguish the two crystal structures. This phenomenon is optical activity, and it is found in natural organic substances such as sugars, camphor, or tartaric acid, as well as in inorganic materials such as low quartz and tellurium. Many optically active molecules or crystals have enantiomers, or stereoisomers, whose atomic configurations are exact mirror images of each other and are thus handed, like our right and left hands. This geometrical property of crystals and molecules is called as chirality. While optical activity distinguishes between right and lefthanded crystals, it does not identify the absolute structure as right or left handed.

The determination of the absolute structure of enantiomers is not an easy experiment because the enantiomers have exactly the same chemical formula and atom to atom arrangements, and is hence considered an important experimental challenge of crystallography. Among many methods developed for the determination so far, $\mathrm{x}$-ray diffraction with dispersion corrections has played an important role for a long time. The scattering length of $x$-rays for an atom can be written as

$$
f=f_{0}+f^{\prime}+i f^{\prime \prime},
$$

where $f_{0}$ is the energy-independent $\mathrm{x}$-ray scattering length and corresponds to the Fourier transform of the electron density around the atom, $f^{\prime}$ and $f^{\prime \prime}$ are the real and the imaginary parts, respectively, of the dispersion correction. It is wellknown that these correction terms depend on the wavelength or the energy of x-rays. Early studies using a fixed photon energy showed that $\mathrm{x}$-ray diffraction using dispersion corrections is useful to determine the absolute structure of $\mathrm{ZnS} .^{1,2}$ Specifically, it was shown that the atomic arrangements along the [111] direction has polarity, i.e., one could distinguish specific surface terminations of $\mathrm{ZnS}$ using $\mathrm{x}$-ray diffraction. It was found that the dull and stepped surface (111) with triangular marks consists of $\mathrm{Zn}$ atoms only whereas the smooth and shiny surface $(\overline{1} \overline{1})$ consists of $\mathrm{S}$ atoms only. The method was also applied to solve the absolute configuration of tartaric acid and carbohydrates by Bijvoet in $1951 .^{3}$ Later, using multiple wavelengths (energies) across an absorption edge to achieve resonant scattering, this method was extensively developed to study the absolute configurations for biochemical compounds.

Since the advent of the synchrotron radiation source, the role of linear polarization of the incident beam, with and without polarization analysis of the scattered beam, has been recognized. Such studies revealed important aspects and applications of magnetic and nonmagnetic resonant scattering. Pioneering studies by Templeton and Templeton ${ }^{4,5}$ and by Dmitrienko et al. ${ }^{6}$ led to the field of anisotropy tensor of $\mathrm{X}$-ray susceptibility to describe the resonant $\mathrm{x}$-ray diffraction. There are excellent reviews now in the literature which have summarized recent advances in the field. ${ }^{7,8}$

In a recent study, we have developed a resonant x-ray diffraction technique using circularly polarized incident $\mathrm{X}$-rays to investigate the structure of chiral crystals. We could clearly show that circularly polarized x-rays can differentiate the crystal chirality by coupling to the crystal screw axis in low quartz, ${ }^{9,10}$ even without polarization analysis of the scattered beam. However, the results are not sufficient to determine the absolute structural chirality by themselves because 
the mixture of two resonant processes gives a complicated theoretical formula and prevents us from determining the sign of chirality. Note that the fundamental concepts about "the absolute structure" and "the absolute configuration," and their determination have been well discussed and defined in crystallography, for example, in Refs. 11 and 12. Here we use the term "absolute structural chirality" to specify the sign of chirality in the space group which have the screw axes labeled right-handed $\left(3_{1}, 4_{1}, 6_{1}\right.$, and $\left.6_{2}\right)$ and left-handed $\left(3_{2}\right.$, $4_{3}, 6_{5}$, and $6_{4}$ ), i.e., for a single crystal with an a priori unknown handedness, it determines whether the crystal is right handed or left handed.

In the present paper, we demonstrate that circularly polarized $\mathrm{x}$-rays provide a sufficient and elegant tool to determine the absolute sign of chirality of a crystal from resonant diffraction described by an $E 1 E 1$ process. We present experimental results of resonant $\mathrm{x}$-ray diffraction from a berlinite crystal $\left(\mathrm{AlPO}_{4}\right)$, compare it with results from low quartz $\left(\mathrm{SiO}_{2}\right)$, and show both results provide unambiguous evidence for determining the structural chirality of the crystals.

\section{ATOMIC STRUCTURE}

Berlinite $\left(\mathrm{AlPO}_{4}\right)$ has the same atomic structure as quartz, obtained by replacing $\mathrm{Si}$ with $\mathrm{Al}$ and $\mathrm{P}$ atoms alternatively along the $c$ axis. Accordingly, berlinite has chirality described by two different space groups $P 3_{1} 21$ (\#152 righthanded screw) and $P 3_{2} 21$ (\#154 left-handed screw). These space groups are mirror images of each other. Hereafter we use the terms $R$ quartz and $R$ berlinite for the crystal structure which belongs to the space group $P 3_{1} 21$ and $L$ quartz and $L$ berlinite for the crystal structure which belongs to the space group $P 3_{2} 21$. The atomic configurations of $R$ quartz and $L$ berlinite are shown in Fig. 1. Note that the alternative replacement of $\mathrm{Si}$ atoms of quartz with $\mathrm{Al}$ and $\mathrm{P}$ atoms changes the sign of chirality together with the space group, and doubles the lattice constant along the $c$ axis, and that the difference between space group $P 3_{1} 21$ and $P 3_{2} 21$ is simply due to the stacking sequence of atomic planes along the $c$ axis.

Another important point to note is the relation between the space groups and optical activity. It has been known that $R$ quartz and $L$ berlinite has the same laevorotatory optical activity, in spite of the fact that their signs of chirality in terms of the space groups are opposite to each other. This is simply because the sign of the optical rotatory power is governed by the oxygen atoms in quartz and berlinite crystals and the oxygen atom positions in $R$ quartz and $L$ berlinite are the same. ${ }^{13}$ Conversely, the measurement of optical rotatory power is not directly sensitive to the structural chirality and cannot be used to determine the sign of structural chirality or space group. As we show in this work, determination of the absolute structure can be achieved by microscopic methods such as X-ray diffraction or neutron diffraction.

\section{EXPERIMENT}

\section{A. Resonant $x$-ray diffraction}

The general diffraction geometry is illustrated in Fig. 2. The scattering plane is defined by vectors $\mathbf{k}_{i}$ and $\mathbf{k}_{f}$, which
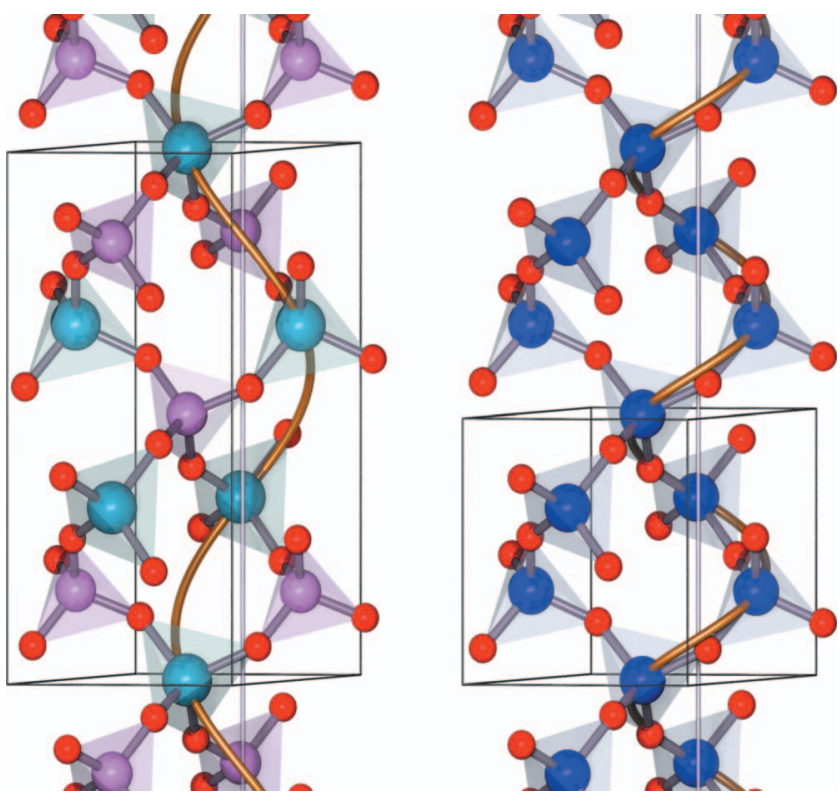

FIG. 1. (Color) Atomic structures of $R$ quartz (right panel) and $L$ berlinite (left panel). Blue and red spheres represent $\mathrm{Si}$ and $\mathrm{O}$ atoms, respectively in quartz, and light blue, pink, and red spheres represent $\mathrm{Al}, \mathrm{P}$, and $\mathrm{O}$ atoms, respectively in berlinite. A white line in each panel is one of the threefold screw axes and a screw line is a guide to eyes. Black lines show the unit cell for each crystal.

are the propagation vectors of the incident and diffracted beams, respectively. The $\sigma$ and $\sigma^{\prime}$ are linear components of $\mathrm{X}$-rays perpendicular to the scattering plane for the incident and diffracted beam, respectively. Their Stokes parameters, ${ }^{14,15}$ which represent the polarization of $\mathrm{X}$-ray beam, are $P_{3}=+1$. Similarly, $\pi$ and $\pi^{\prime}$ are linear components of $\mathrm{x}$-rays parallel to the scattering plane for the incident and diffracted beam, respectively, and their stokes parameters are $P_{3}=-1$. Here $\sigma\left(\sigma^{\prime}\right), \pi\left(\pi^{\prime}\right)$, and $\mathbf{k}_{i}\left(\mathbf{k}_{f}\right)$ has a right-handed relation $\left(\hat{\sigma} \times \hat{\pi}=\hat{\mathbf{k}}_{\mathbf{i}}\right.$ and $\left.\hat{\sigma}^{\prime} \times \hat{\pi}^{\prime}=\hat{\mathbf{k}}_{\mathbf{f}}\right)$ as the $x, y$, and $z$ axes has a right-handed coordinate system $\hat{x} \times \hat{y}=\hat{z})$. Each hat denotes the unit vector. The positive (clockwise) and negative (counterclockwise) circularly polarized x-rays are expressed in terms of the helicity, $P_{2}$, and with our convention, $P_{2}=+1$ and -1 , respectively. The positive (negative) circularly polarized x-rays are expressed by a sum of $\hat{\sigma}$ and $\hat{\pi}$

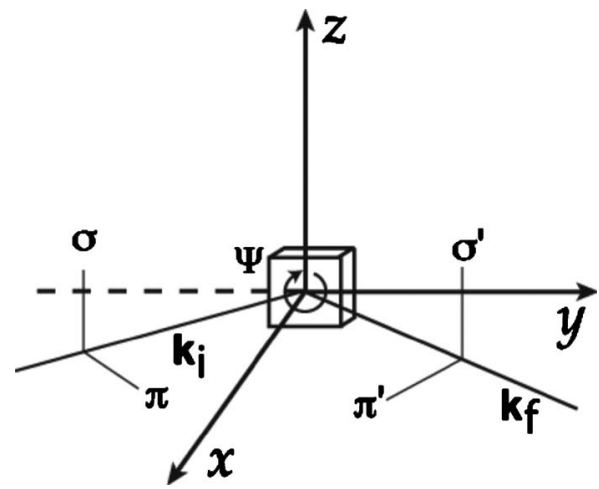

FIG. 2. A schematic view for Bragg diffraction with a righthanded coordinates $x, y$, and $z$. 

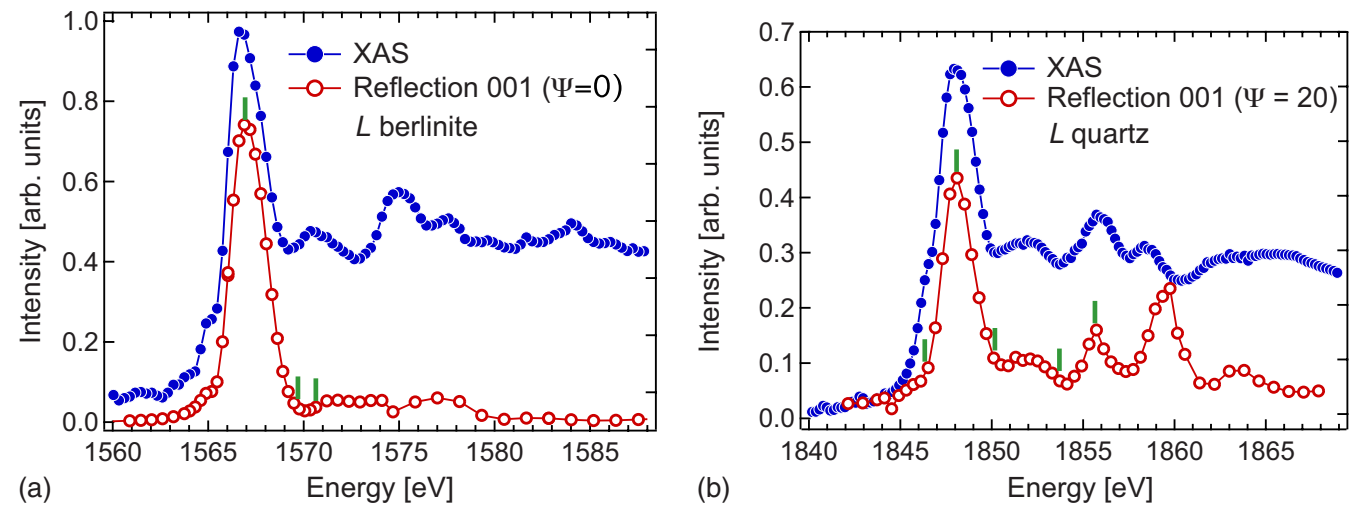

FIG. 3. (Color online) (a) XAS (filled circles) obtained by the total fluorescence yield of $L$ berlinite in the vicinity of Al $1 s$ absorption edge and the integrated intensity (red open circles) of reflection 001 measured at azimuth angle $\Psi=0^{\circ}$ as a function of energy of the $(+)$ helicity incident beam. (b) XAS and the integrated intensity of reflection 001 of $L$ quartz measured at $\Psi=20^{\circ}$ as a function of energy of the $(+)$ helicity incident beam (adopted from Ref. 9). Small bars in each panel correspond to the energies at which the azimuth angle scans are performed. They are shown in Figs. 5 and 6.

linear polarization in which the $\hat{\sigma}$ component is advanced (behind) in time by a phase $\frac{\pi}{2}$ to the $\hat{\pi}$ component, and the spatial trace of the electric field of the positive (negative) circularly polarized x-rays at any instant of time is a left (right)-handed screw, respectively. Note that the polarization of the beam from the synchrotron radiation source is usually well defined and that the relation $P_{1}^{2}+P_{2}^{2}+P_{3}^{2} \cong 1$ holds. The azimuthal angle $\Psi$ is a rotation of the sample about the scattering vector $\mathbf{k}=\mathbf{k}_{f}-\mathbf{k}_{i}$; opposite sign to Ref. 10 and the positive direction is clockwise as viewed looking up along the scattering vector $\mathbf{k}$.

Experiments were carried out at the beamline $17 \mathrm{SU}$ at SPring-8 in Harima, Japan. ${ }^{16}$ The incident energy was tuned in the vicinity of $\mathrm{Al} 1 \mathrm{~s}$ absorption edge around $E$ $=1567 \mathrm{eV}$ by the grating apparatus. The focused spot size at the sample was about $10 \mu \mathrm{m} \times 10 \mu \mathrm{m}$. The helicity of the incident beam was switched by the electromagnet of the undulator. ${ }^{17}$ The Stokes parameters calculated from the insertion device parameters at $E=1567 \mathrm{eV}$ are given by $P_{2}=+0.95$ and $P_{3}=+0.30$ and $P_{2}=-0.95$ and $P_{3}=+0.30$ for the positive $(+)$ and negative $(-)$ helicity of the circularly polarized incident beam, respectively. In the previous experiment on low quartz, ${ }^{9}$ we used an incident beam energy of $E=1847.9 \mathrm{eV}$ and the Stokes parameters were $P_{2}=+0.95$ and $P_{3}=-0.31$, and $P_{2}=-0.95$ and $P_{3}=-0.31$ for the positive $(+)$ and negative $(-)$ helicity of the incident beam, respectively. The value of $P_{3}$ (degree of linear polarization) is unchanged by switching the helicity of the incident beam. The photon flux measured with a photosensitive diode for $E$ $=1567 \mathrm{eV}$ and for $E=1848 \mathrm{eV}$ is about 1.8 $\times 10^{10}$ photons $/ \mathrm{sec}$ and $1.0 \times 10^{10} \mathrm{photons} / \mathrm{sec}$, respectively. The energy resolution for $E=1567 \mathrm{eV}$ (Al $1 s$ absorption edge) and for $E=1848 \mathrm{eV}$ ( $\mathrm{Si} 1 s$ absorption edge) is about $0.3 \mathrm{eV}$ and $0.4 \mathrm{eV}$, respectively.

The intensity of the diffracted beam was measured by a photosensitive diode, without a polarization analysis system. Samples were mechanically polished and mounted on the sample stage of the diffractometer which has two rotational axes for alignment. The azimuth axis $\Psi\left(-95^{\circ}<\Psi<95^{\circ}\right)$ enables us to measure the intensity as a function of the angle rotated about the scattering vector of the crystal. The second rotation axis is used for tilt $\chi$ motion. Details of the diffractometer are reported in Ref. 18.

\section{B. Optical rotatory power}

We have measured the optical rotatory power of the berlinite and quartz samples using a He-Ne laser system (wavelength $\lambda=533 \mathrm{~nm}$ ). The thickness of each sample is $1 \mathrm{~mm}$. The berlinite sample shows a rotatory power of $\gamma=-14 \pm 1$ degrees $/ \mathrm{mm}$, i.e., a laevorotatory power (counterclockwise rotation observing toward the laser source), indicating that the sample belongs to the space group $P 3_{2} 21$ according to Ref. 19. Hence, our berlinite sample is $L$ berlinite. We also confirmed that our quartz samples of $R$ quartz and $L$ quartz show that $\gamma=-18 \pm 1$ and $+18 \pm 1$ degrees $/ \mathrm{mm}$ of rotatory power, respectively. ${ }^{13}$ The sign + corresponds to the clockwise direction. In this paper, we show the experimental results on $L$ berlinite and $L$ quartz.

\section{RESULTS}

\section{A. X-ray absorption spectrum}

Figure 3 shows the $\mathrm{x}$-ray absorption spectrum (XAS) obtained in the total $\mathrm{X}$-ray fluorescence yield mode of $L$ berlinite together with that of $L$ quartz in the vicinity of $\mathrm{Al}(\mathrm{Si}) 1 s$ absorption edge and the integrated intensity of the spacegroup forbidden reflection 001 as a function of energy of the (+) helicity incident beam. In principle, natural circular dichroism (NCD) is allowed in quartz and berlinite and we have checked for the same. However, we could not find any signature of NCD in our XAS measurements. The effect may be too small $\left(<10^{-3}\right)$ to be observed. On the other hand, for example, it is reported that clear NCD signal has been observed in $\mathrm{CsCuCl}_{3}$. ${ }^{20}$

The measured XAS of berlinite at the $\mathrm{Al} 1 s$ and $\mathrm{Si} 1 s$ show very similar features. They have a sharp peak at the Al (Si) $1 s$ absorption edge, and some small bumps at higher energies. For both the samples, the 001 diffraction intensity for $L$ berlinite and $L$ quartz show a sharp peak corresponding 


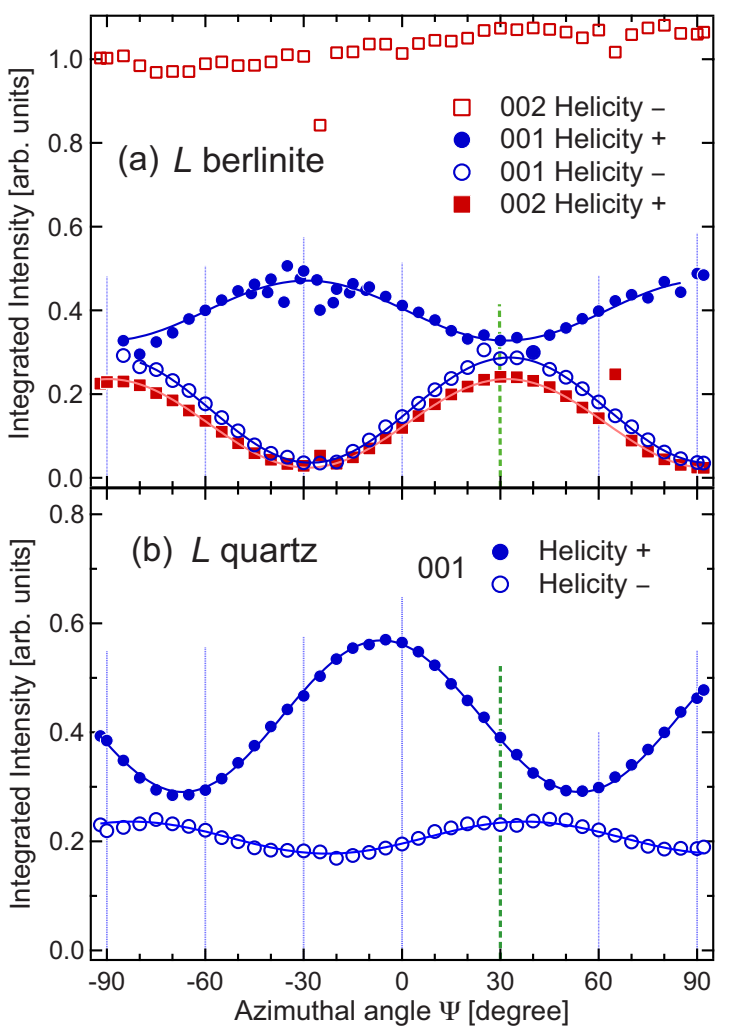

FIG. 4. (Color online) (a) Integrated intensity of reflection 001 and 002 as a function of azimuthal angle. Filled circles (squares) represent the intensity of reflection 001 (002) of $L$ berlinite measured with the incident beam with $(+)$ helicity and open circles (squares) represent the intensity of reflection 001 (002) measured with (-) helicity. (b) (Adopted from Ref. 9) Integrated intensity of reflection 001 of $L$ quartz as a function of azimuthal angle. Filled and open circles represent the intensity of reflection 001 of $L$ quartz measured with the incident beam with (+) helicity and (-) helicity, respectively. Each line shows a result of fit to data with functions expressed by Eq. (6) for panel (a) and Eq. (9) for panel (b). The dashed vertical lines at $\Psi=30$ show the position where the direction of the direct and diad (twofold) lattice axis $a$ is parallel to the $z$ axis.

to the highest intensity peak of the XAS spectra, and have weaker bumps at higher energies. The relative intensity of the weaker spectral features is more prominent for quartz than for berlinite. We will discuss this later. Most importantly, for either case, the 001 diffraction intensity becomes negligible as the incident energy is tuned away from the $\mathrm{Al}$ (Si) $1 \mathrm{~s}$ absorption edge, just below and about $15 \mathrm{eV}$ above the main peak.

\section{B. Azimuthal angle diffraction scans}

We have first measured the integral intensity of reflections 001 and 002 of $L$ berlinite and reflection 001 of $L$ quartz as a function of the azimuth angle $\Psi$ at the main resonance energy and by switching the helicity of the incident beam. Figure 4 shows the azimuth scans for $L$ berlinite observed at the resonant energy $E=1566.8 \mathrm{eV}$, where the intensity of the diffracted beam is the maximum, together with the data for $L$ quartz measured at $E=1847.9 \mathrm{eV}$. The data are corrected for

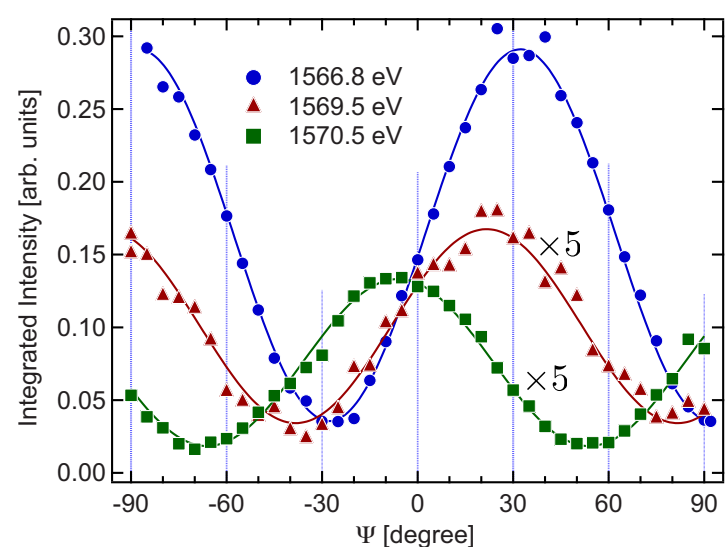

FIG. 5. (Color online) Integrated intensity of reflection 001 of $L$ berlinite as a function of azimuthal angle. Filled circles, triangles, and squares represent the intensity measured with the incident beam of (-) helicity at $E=1566.8 \mathrm{eV}, 1569.5$, and $1570.5 \mathrm{eV}$, respectively. The scan data measured at $E=1569.5 \mathrm{eV}$ and $1570.5 \mathrm{eV}$ are multiplied by a factor of 5 so as to easily compare with the scan measured at $E=1566.8 \mathrm{eV}$.

the absorption and Lorentz factors. Here, the origin of the azimuth angle $\Psi=0$ is defined with respect to the direction of the reciprocal lattice vector $a^{*}$ axis when it is parallel to the $z$ axis in Fig. 2.

We find the following main features in Fig. 4 for $L$ berlinite: (i) each azimuth scan [except the scan of reflection 002 for the (-) helicity beam, which is almost flat], shows a threefold sinusoidal curve which is symmetric in terms of $\Psi=30$. (ii) the data of reflection 001 for the (+) helicity beam and that for the $(-)$ helicity beam exhibit an antiphase relation with each other as a function of $\Psi$, (iii) the intensity of reflection 001 is higher for the $(+)$ helicity beam than for the (-) helicity beam and this feature is observed for $L$ quartz as well, (this relation is opposite for $R$ quartz) and (iv) the relation of the intensity of reflection 002 between for $(+)$ and (-) helicity beams is opposite to that of reflection 001 . Thus, the diffracted intensities show evidence of a strong correlation between the helicity of the incident beam and the crystal chirality. Similar evidence for a strong correlation between the incident beam helicity and crystal chirality was reported by us for quartz..$^{9,10}$

We next discuss the energy dependence of azimuth scans for the 001 reflections of $L$ berlinite and $L$ quartz. The azimuth scans at several energies (energy positions are marked in right and left panels of Fig. 3, respectively) of $L$ berlinite measured with the (-) helicity beam and those of $L$ quartz measured with both helicity beams are shown in Figs. 5 and 6. The profiles shown in Figs. 5 and 6 indicate that the phase of azimuth scans changes with the energy of the incident beam. As is observed, while the phase shifts as a function of energy, each azimuth scan retains a threefold sinusoidal curve in accord with the crystal symmetry. In the following, we analyze the presented data to clarify the role of $E 1 E 1$ transition in explaining the crystal chirality of $L$ berlinite at the main peak resonance, and the importance of an E1E2 event in giving rise to the phase shifts as a function of energy. We also discuss the energy dependence of the phase 


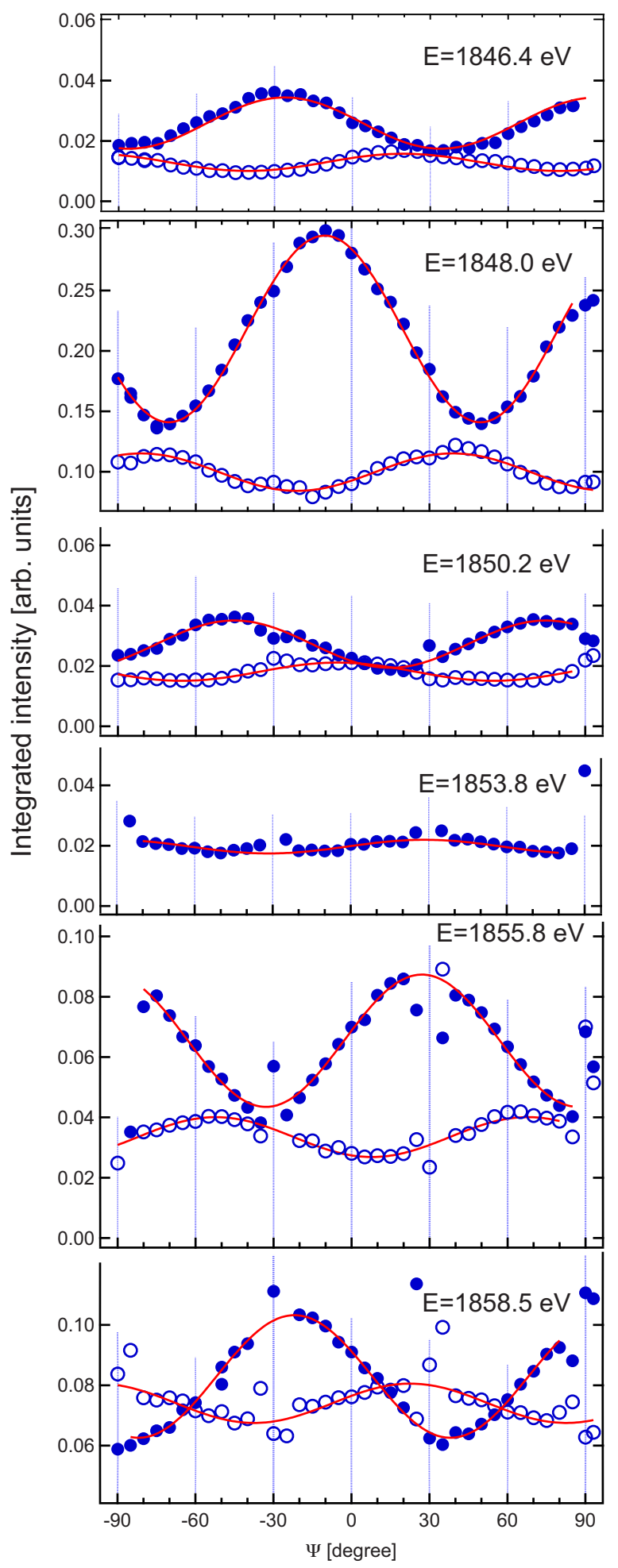

FIG. 6. (Color online) Integrated intensity of reflection 001 of $L$ quartz as a function of azimuthal angle and energy of the incident beam. Filled and open circles represent the intensity measured with the incident beam of (+) helicity and (-) helicity, respectively.

shift in $L$ quartz, but for $L$ quartz evidently, the azimuth scans for the space-group forbidden reflections cannot be explained by a single $E 1 E 1$ event even for the main resonance. ${ }^{9,10}$

\section{ANALYSIS}

\section{A. Resonant diffraction of circularly polarized $x$-rays}

The intensity $I$ of the resonant diffraction is described using the Stokes parameters $\left(P_{1}=0, P_{2}, P_{3}\right), P_{2}=$ helicity, as

$$
\begin{aligned}
I= & \frac{1}{2}\left(1+P_{3}\right)\left(\left|G_{\sigma^{\prime} \sigma}\right|^{2}+\left|G_{\pi^{\prime} \sigma}\right|^{2}\right)+\frac{1}{2}\left(1-P_{3}\right) \\
& \times\left(\left|G_{\pi^{\prime} \pi}\right|^{2}+\left|G_{\sigma^{\prime} \pi}\right|^{2}\right)+P_{2} \operatorname{Im}\left(G_{\sigma^{\prime} \pi}^{*} G_{\sigma^{\prime} \sigma}+G_{\pi^{\prime} \pi}^{*} G_{\pi^{\prime} \sigma}\right) .
\end{aligned}
$$

This equation is readily derived either following the discussion of polarization in Ref. 15, or immediately from Eqs. (24) and (26) in Ref. 8. Here $G_{\mu^{\prime} \nu}$ is the total resonant scattering amplitude, and $\mu^{\prime}$ and $\nu$ are the polarization state of the diffracted and incident $\mathrm{x}$-ray beam, respectively. The first term is the $\sigma$ channel term, the second term is the $\pi$ channel term and the third term represents the interference between $\sigma$ and $\pi$ components in the resonant scattering process for the circularly polarized x-rays. The third term plays a crucial role for determination of the chirality by changing the sign coupling with the helicity. This interference term does not appear in conventional diffraction (Thomson scattering) even with the circularly polarized x-ray beam because there are no events which change the polarization state, i.e., resonant scattering amplitudes $G$ (and its complex conjugates $G^{*}$ ) of the type $G_{\pi^{\prime} \sigma}=G_{\sigma^{\prime} \pi}=0$.

When the resonant process has several events,

$$
G_{\mu^{\prime} \nu}=\sum_{k} r^{(k)} \frac{F_{\mu^{\prime} \nu}^{(k)}}{E-\Delta_{k}+\frac{i}{2} \Gamma_{k}}=\sum_{k} d^{(k)}(E) F_{\mu^{\prime} \nu}^{(k)},
$$

where $k$ represents individual resonant events like $E 1 E 1$, $E 1 E 2$ etc., $\Delta_{k}, \Gamma_{k}$, and $r^{(k)}$ represent the resonant energy, its width, and the mixing parameter, respectively, and the scattering amplitude $F_{\mu^{\prime} \nu}$ is

$$
F_{\mu^{\prime} \nu}=\sum_{K} X_{\mu^{\prime} \nu}^{(K)} D^{(K)} \Psi^{(K)} .
$$

In this expression, $\Psi^{(K)}$ represents the unit-cell structure factor tensor of rank $K$ which is the sum of atomic multipoles related to the resonant process and $X_{\mu^{\prime} \nu}^{(K)}$ describes conditions of the incident and the diffracted beam. Orientation of the crystal, with respect to states of polarization and the plane of scattering, is accomplished by a rotation matrix $D^{(K)}$. The theoretical formalism is described in detail in Ref. 8.

\section{B. Structure factor}

The structure factor for the enantiomorphic space-group pair \#152 (right-handed screw) and \#154 (left-handed screw) is fully described in Ref. 10. Here we describe the structure factor and the intensity for circularly polarized x-rays briefly. As described in Sec. II, the difference between the two space groups is on the stacking sequence of atomic planes along the $c$ axis. $\mathrm{Al}$ and $\mathrm{Si}$ atoms locate at $(-x,-x, 0),\left(x, 0, \pm \frac{1}{3}\right)$, and $\left(x, 0, \mp \frac{1}{3}\right)$ in a unit cell of berlinite and quartz, respec- 
TABLE I. Inferred values of $I_{0}$ and $I_{1}$ in Eq. (6) and the experimental coefficients $\left(I_{0}^{c}\right.$ and $\left.I_{1}^{c}\right)$ of $I_{0}$ and $I_{1}$.

\begin{tabular}{cccccc}
\hline \hline Reflection & Helicity & $I_{0}$ & $I_{0}^{c}$ & $I_{1}$ & $I_{1}^{c}$ \\
\hline 001 & $P_{2}=+0.95$ & 0.399 & $1.17 T_{a}^{2}+0.87 T_{b}^{2}$ & -0.071 & $-0.57 T_{a} T_{b}$ \\
001 & $P_{2}=-0.95$ & 0.162 & $0.40 T_{a}^{2}+0.87 T_{b}^{2}$ & 0.125 & $0.97 T_{a} T_{b}$ \\
002 & $P_{2}=+0.95$ & 0.130 & $0.22 T_{a}^{2}+0.48 T_{b}^{2}$ & 0.105 & $0.61 T_{a} T_{b}$ \\
002 & $P_{2}=-0.95$ & 1.030 & $2.31 T_{a}^{2}+0.48 T_{b}^{2}$ & -0.01 & $-0.01 T_{a} T_{b}$ \\
\hline \hline
\end{tabular}

tively. Here the upper and lower sign of $\frac{1}{3}$ represent the space-groups \#152 and \#154, respectively. We keep this sign notation for the following discussion.

The most probable resonant process at the main peak of the absorption edges of $\mathrm{Al}$ and $\mathrm{Si}$ shown in panels (a) and (b) of Fig. 3 is the $E 1 E 1$ event $(1 s \leftrightarrow 3 p)$. The atomic multipole related to the $E 1 E 1$ event is $\left\langle T_{Q}^{(K)}\right\rangle$. Here rank $K$ and its projection $Q$ satisfy $K=2$ and $-K \leq Q \leq K$. We take the ion at site $(-x,-x, 0)$ as a reference and describe the atomic multipole $\left\langle T_{Q}^{(2)}\right\rangle$ with a right-handed orthogonal quantization axes $(\xi \eta \zeta)$. Here $\xi$ axis coincides with the diad (twofold) axis of rotation symmetry through $(-x,-x, 0)$ and $\zeta$ axis coincides with the crystal $c$ axis. Note that our $\xi$ axis lies in the plane spanned by reciprocal lattice vectors $\mathbf{a}^{*}$ (normal to direct vectors $\mathbf{b}$ and $\mathbf{c}$ ) and $\mathbf{b}^{*}$, and $\mathbf{a}^{*}$ and the $\xi$ axis enclose an angle of $30^{\circ}$ and that the direct vectors $\mathbf{a}, \mathbf{b}$, and $\mathbf{c}$ as well as the reciprocal vectors $\mathbf{a}^{*}, \mathbf{b}^{*}$, and $\mathbf{c}^{*}$ make a right-handed coordinate system.

The unit-cell structure factor for \#152 and \#154, and reflection $00 l(l>0)$ is found to be

$$
\Psi_{Q}^{(2)}=\left\langle T_{Q}^{(2)}\right\rangle\left\{1+\mathrm{e}^{2 \pi i Q / 3} \mathrm{e}^{2 \pi i( \pm l / 3)}+\mathrm{e}^{-2 \pi i Q / 3} \mathrm{e}^{2 \pi i(\mp l / 3)}\right\} .
$$

Here we find the selection rule for reflection $00 l$ that $l+Q$ $=3 n$ for space-group $\# 152$ and $l-Q=3 n$ for space-group $\# 154$, where $n$ is an integer. Space group allowed reflections satisfy the condition $Q=0$ and $l=3 n$. Note that Eq. (5) appears to suggest that reversing the sign of $l$ is equivalent to reversing $Q$, and thus the helicity. However, it should be remembered that such a reversal also requires a transformation of the tensor $T_{Q}^{(K)}$. The resulting scattering amplitude does not respect the apparent connection between the sign of $l$ and the helicity of the crystal.

Accordingly the structure factors for reflection 001 for space-groups \#152 and \#154 are represented by a sum of $3\left\langle T_{-1}^{(2)}\right\rangle$ and $3\left\langle T_{+2}^{(2)}\right\rangle$ and a sum of $3\left\langle T_{+1}^{(2)}\right\rangle$ and $3\left\langle T_{-2}^{(2)}\right\rangle$, respectively. It is interesting to note that the relation between the structure factor and the space group is interchanged for reflection 002. This is because the phase factor in the calculation of the structure factor for reflection 001 and reflection 002 is opposite to each other. Following the same procedures written in Ref. 10, the intensity of $00 l(l \neq 3 n, n$ is an integer) for space-groups \#152 and \#154 for the $E 1 E 1$ resonant event is found to be

$$
I=I_{0}+I_{1} \cos \{3(\Psi-30)\}
$$

$$
\begin{aligned}
& I_{0}= \cos ^{2} \theta T_{b}^{2}+\frac{1+\sin ^{2} \theta}{2} \\
& \times\left\{1+\sin ^{2} \theta+P_{3} \cos ^{2} \theta-2 \nu P_{2} \sin \theta\right\} T_{a}^{2}, \\
& I_{1}=\left\{2 P_{3} \sin \theta+\nu P_{2} \cos ^{2} \theta\right\} \cos \theta T_{a} T_{b} .
\end{aligned}
$$

Here $I_{0}$ and $I_{1}$ are constants for a particular Bragg angle $\theta$, and the coefficient of $I_{1}$ in Eq. (6) is the cosine term which gives azimuth dependence. Also, $T_{a}=\frac{3}{2}\left\langle T_{+2}^{(2)}\right\rangle^{\prime}$, and $T_{b}$ $=\frac{3}{2}\left\langle T_{+1}^{(2)}\right\rangle^{\prime \prime}$ (the single prime and the double prime represent the real and the imaginary parts of each atomic multipole, respectively), $\Psi$ is the experimental azimuth angle and is 30 degrees off from the origin (shown in both panels of Fig. 4 as a dashed line) for the theoretical calculations. We introduce a parameter $\nu= \pm 1$ to label the chirality, the sign of which depends on the Miller indices $00 \mathrm{l}$ as well. From a consistent analysis of the data, we show in the following that for the 001 reflection, $\nu=+1$ for space-group \#152 and $\nu=-1$ for space-group \#154 and moreover, the sign is reversed for the 002 reflection. Note that the sign of the $P_{2}$ terms in Eqs. (7) and (8) is opposite to those in the equations described in Ref. 10 since we have changed the definition of the scattering vector to be $\mathbf{k}=\mathbf{k}_{f}-\mathbf{k}_{i}$.

\section{DISCUSSION}

Let us first analyze the azimuth scans of $L$ berlinite shown in Fig. 4 using Eqs. (6)-(8). Here experimental parameters $\theta$, $P_{2}$, and $P_{3}$ are known: $\sin \theta=0.361$ for reflection 001 and $\sin \theta=0.722$ for reflection $002, P_{2}= \pm 0.95$, and $P_{3}=+0.30$. Parameters $T_{a}$, and $T_{b}$, and $\nu$ are unknown. As shown in Fig. 4 , all scans can be nicely fitted with a function expressed by Eq. (6), except for reflection 002 with (-) helicity. Some isolated points show deviations from the curves and these are attributed to multiple scattering.

From the fit results to the azimuth scans carried out using Eq. (6), we list the obtained parameters $I_{0}$ and $I_{1}$ in columns 3 and 5 of Table I. Using Eq. (7), we calculate the corresponding values of the coefficients $T_{a}^{2}$ and $T_{b}^{2}$. Here we use the experimental parameters (Bragg angle $\theta$ and Stokes parameters $P_{2}$, and $P_{3}$ ) together with the parameter $\nu$ as discussed below. The values of coefficients are listed in column 4 of Table I. Then, we obtain the values of $T_{a}^{2}$ and $T_{b}^{2}$. For reflection 001, there are two equations, $I_{0}(+)=0.399$ $=1.17 T_{a}^{2}+0.87 T_{b}^{2}$ and $I_{0}(-)=0.162=0.40 T_{a}^{2}+0.87 T_{b}^{2}$. These give the values of $T_{a}^{2}=0.306$ and $T_{b}^{2}=0.045$.

First, the change in $I_{0}$ for $(+)$ and $(-)$ helicity, $I_{0}(+)$ $-I_{0}(-)=-0.95 \times 2\left(1+\sin ^{2} \theta\right) \sin \theta \nu T_{a}^{2},\left(P_{2}= \pm 0.95\right)$, can be 
TABLE II. Values of atomic multipoles of $T_{a}$ and $T_{b}$ obtained from the fit.

\begin{tabular}{|c|c|c|c|c|c|}
\hline \multirow[b]{2}{*}{ Reflection } & \multicolumn{3}{|c|}{$I_{0}$} & \multirow{2}{*}{$\begin{array}{c}I_{1}, P_{2}=+0.95 \\
T_{a} T_{b}\end{array}$} & \multirow{2}{*}{$\begin{array}{c}I_{1}, P_{2}=-0.95 \\
T_{a} T_{b}\end{array}$} \\
\hline & $T_{a}^{2}$ & $T_{b}^{2}$ & $T_{a} T_{b}$ & & \\
\hline 001 & 0.306 & 0.045 & 0.117 & 0.125 & 0.128 \\
\hline 002 & 0.430 & 0.072 & 0.176 & 0.171 & \\
\hline
\end{tabular}

used to unambiguously determine the parameter $\nu$ for both reflections 001 and 002 . Since the values of atomic multipole parameters $T_{a}$ and $T_{b}$ are real numbers, for the 001 reflection, the higher intensity of $I_{0}$ with $(+)$ helicity and the lower intensity with $(-)$ helicity directly indicates $\nu=-1$. In contrast, the opposite assignment of $\nu=+1$ is incompatible with the 001 reflection data. The same exercise is then repeated for the 002 reflection. In particular, the large increase in intensity of the 002 reflection with $(-)$ helicity and smaller intensity for $(+)$ helicity, is compatible only with $\nu=+1$, although, as discussed later, we could not obtain a reliable fit to the azimuth scan with (-) helicity due to the small variations in intensity as a function of azimuth angle $\Psi$.

Next, using Eq. (8), we can accordingly calculate the values of the coefficient of $T_{a} T_{b}$ in column 6 of Table I from the experimental parameters together with the parameter $\nu$ as discussed below. The absolute values of $I_{1}$ for (+) and (-) helicity can be used to unambiguously determine the parameter $\nu$ for both reflections 001 and 002, too, and independently of the above argument on $I_{0}$. Compare columns 5 and 6 in Table I. Clearly, reflection 001 has the lower absolute value of $I_{1}$ for $(+)$ helicity than $(-)$ helicity and reflection 002 has the higher absolute value $I_{1}$ for $(+)$ helicity than $(-)$ helicity. Hence, along with the above discussion on $I_{0}$, we find the chirality parameter $\nu=-1$ for reflection 001 and $\nu$ $=+1$ for reflection 002. The opposite assignment of $\nu$ is incompatible with the data. Thus, the result clearly shows that the berlinite crystal we measured belongs to the space group $P 3_{2} 21$, \#154 but not to $P 3_{1} 21$, \#152. Note that this result is independent of the measurement of optical rotatory power.

Let us discuss the values of atomic multipole parameters $T_{a}$ and $T_{b}$. We use the chirality parameter $\nu=-1$ for reflection 001 and $\nu=+1$ for reflection 002 , and then obtain the values of $T_{a}^{2}$ and $T_{b}^{2}$ independently from $I_{0}$ of reflection 001 and 002 , as listed in Table II. Moreover, the values of $T_{a} T_{b}$ are obtained independently from the fit results of $I_{1}$ for both positive and negative helicity.

The constant term $I_{0}$ for reflection 001 and for reflection 002 give the quite similar values of relative magnitudes for $T_{a}^{2}$ and $T_{b}^{2}$, independently. The values $T_{a}^{2}$ and $T_{b}^{2}$ obtained from reflection 001 are 0.306 and 0.045 , respectively, while those obtained from reflection 002 are 0.430 and 0.072 , respectively. The ratio $T_{a}^{2} / T_{b}^{2}$ for reflection 001 is 6.8 and that for reflection 002 is 5.9 .

Moreover the amplitude term $I_{1}$ of scans for the positive and negative helicity of reflection 001 , as well as the scan for the positive helicity of reflection 002 give almost the same values for $T_{a} T_{b}$, independently. These values are also consistent with the values of $\left(T_{a}^{2} T_{b}^{2}\right)^{1 / 2}$ obtained from $I_{0}$, which are listed in column 4 in Table II. The values of $T_{a}^{2}, T_{b}^{2}$, and $T_{a} T_{b}$ are slightly higher for reflection 002 than those for reflection 001. This may be due to the resonant effect of the refractive index. The refractive index for $\mathrm{x}$-rays is $\tilde{n}=1-\delta+i \beta$. For hard x-rays, $\delta \propto \lambda^{2}, \beta$ is negligible, and $\delta$ is of the order of $10^{-6}$ (for example $\delta=1.74 \times 10^{-6}$ for $\mathrm{Al}$ and the Mo $K_{\alpha}$ radiation). ${ }^{21}$ However, at resonant energy, for example Al $1 s$ absorption edge, $\delta$ diverges as $\delta \propto 1 /\left(E^{2}-E_{K}^{2}\right)$ and $\tilde{n}<1 .^{21}$ Here $E$ and $E_{K}$ are the energy of the incident beam and the absorption edge of $\mathrm{Al}$, respectively. Due to the lower Bragg angle $\theta$ for reflection 001 compared with reflection 002 , the volume of the sample probed by the x-ray beam would be reduced for reflection 001 compared to 002. This resonant effect may reduce the intensity of reflection 001 compared to that of reflection 002 .

The $I_{1}$ for reflection 002 and negative helicity is accidentally negligible as observed in Fig. 4 and as expected by the calculated coefficient of $T_{a} T_{b}$ (see Table I). While small fluctuations can be observed in the azimuth scan for the 002 reflection with negative helicity, we could not obtain a reliable fit to the data.

Thus, while the absolute configuration of $L$ berlinite could be determined from the azimuth scans at the main resonance energy of $1566.8 \mathrm{eV}$ shown in Fig. 4, the energy dependent scans of Figs. 5 and 6 indicate an additional energy dependent term. Indeed, it was recently recognized that for an analysis of $R$ quartz and $L$ quartz, Eq. (6) is not enough to describe the azimuth scans as discussed in Refs. 9 and 10. Scans shown in lower panel of Fig. 4 cannot be fitted to Eq. (6) because they are not symmetric in terms of the origin $\Psi=30$. We need to add a third term to Eq. (6). Accordingly, the intensity is described as

$$
I=I_{0}+I_{1} \cos \{3(\Psi-30)\}+I_{2} \sin \{3(\Psi-30)\} .
$$

This additional term does not come from an E1E1 parityeven event but from a parity-odd event such as $E 1 E 2$ or $E 1 M 1$ event. In these events, polar multipoles $\left\langle U_{Q}^{(K)}\right\rangle$ are observable. The explicit expressions are found in Eqs. (6.1) to (6.10) in Ref. 10. For a complete analysis, additional parameters $U_{a}, U_{b}, U_{\alpha}, U_{\beta}$, and the mixing parameter $\eta$ are required. It is noted that inclusion of the parity-odd event also leads to a modification of $I_{0}$ and $I_{1}$ in the first two terms of the total intensity. Here $d^{o}(E) / d^{e}(E)=\exp (i \eta)\left|d^{o}(E) / d^{e}(E)\right|$ and $d^{e}(E)$ and $d^{o}(E)$ are the resonance denominators described in Eq. (3) associated with the parity-even and parityodd events, respectively.

Full analysis of the experimental data is quite difficult because of the number of unknown parameters, however, we extract the important feature for the determination of the chirality neglecting additional terms due to polar multipoles in Eq. (9). The fit parameters which reproduce the experi- 
TABLE III. Inferred values of $I_{0}$ and $I_{1}$ in Eq. (9) and the experimental coefficients $\left(I_{0}^{c}\right.$ and $\left.I_{1}^{c}\right)$ of $I_{0}$ and $I_{1}$ for $L$ quartz.

\begin{tabular}{cccccrr}
\hline \hline \multirow{2}{*}{ Reflection } & Helicity & $I_{0}$ & $I_{0}^{c}$ & $I_{1}$ & $I_{1}^{c}$ & \multicolumn{1}{c}{$I_{2}$} \\
\hline \multirow{2}{*}{001} & $P_{2}=+0.95$ & 4.20 & $1.58 T_{a}^{2}+0.625 T_{b}^{2}$ & -0.44 & $-0.97 T_{a} T_{b}$ & -1.31 \\
001 & $P_{2}=-0.95$ & 2.06 & $-0.02 T_{a}^{2}+0.625 T_{b}^{2}$ & 0.27 & $0.21 T_{a} T_{b}$ & 0.10 \\
\hline \hline
\end{tabular}

mental scans for $L$ quartz shown in lower panel of Fig. 4 are summarized in Table III. Here polarization parameters are $P_{2}= \pm 0.95$ and $P_{3}=-0.31$ and the chirality parameter $\nu=$ -1 as discussed below. The values of $I_{0}$ and $I_{1}$ are a result of fit with a function expressed in Eq. (9). We find that the coefficient of parity-even multipoles, $T_{a}$ and $T_{b}$, in $I_{0}$ and $I_{1}$ in Eq. (9) sufficiently describe the feature of experimental data quite well with the chirality parameter $\nu=-1$. Clearly, the opposite assignment of $\nu=+1$ is incompatible with the data. Thus, the experimental results for the 001 reflection of $L$ quartz agrees with the coefficients obtained from the experimental parameters and $\nu=-1$. Hence, just as for $L$ berlinite, we conclude that the quartz crystal we measured belong to the space-group \#154, independent of the optical rotatory power measurement.

Note that the essential term to determine the chirality is the last term of Eq. (7), which represents the coupling between helicity of the incident beam and chirality of the crystal structure because $T_{a}^{2}>0$ always holds. Equation (8) has a coupling term, too, however, if we consider the case of $P_{3}$ $=0$ and $P_{2}= \pm 1$, then switching the helicity just changes the sign of $I_{1}$. In this case we cannot determine the chirality only from the information of $I_{1}$ because we do not know the sign of either $T_{a}$ or $T_{b}$. However, in our experiment the incident beam does not have $100 \%$ circular polarization and includes some amount of linear polarization $\left(P_{3}=+0.30\right.$ for the $L$ berlinite and $P_{3}=-0.31$ for the $L$ quartz). While such an admixture of linear polarization is usually troublesome, we could use it to advantage. The linear polarization contribution changes the absolute value of $I_{1}$ according to the sign of $P_{2}$ because $P_{3}$ is constant. Thus, it helps us to determine the crystal chirality from the results both of $I_{0}$ and $I_{1}$ in azimuth scans. It is noted that the analysis would be simplified if we can select between pure orthogonal states of linear and circular polarization flexibly and if good optical devices to analyze the polarization state of the diffracted beam are available in an energy range from 1000 to $2000 \mathrm{eV}$.

Another method to determine the chirality from $I_{1}$ is to use a polarization analysis with an appropriate analyzer crystal on the diffracted beam. Actually, it has been used to determine the structural chirality of an elemental crystal tellurium. ${ }^{22}$

As described in Sec. III A, the positive and negative circular polarized x-rays have the spatial trace of the electric field of a left- and right-handed screw, respectively. The intensity of reflection 001 of $L$ berlinite and $L$ quartz is enhanced while that of reflection 002 of $L$ berlinite and $L$ quartz is weakened by the positive circular polarized X-rays which has a left-handed screw in space. Hence, our finding is summarized by a simple sentence, "When the sign of the phase factor of the structure factor in the crystal and that of the spatial trace of the electric field of the circularly polarized $x$-rays coincides, the diffraction intensity is enhanced whereas in the other case, the intensity is diminished."

Finally, it is noted that our results do provide evidence of the mixture of parity-odd resonant events with the $E 1 E 1$ event in the form of an energy dependent phase shift in Fig. 5 for $L$ berlinite and Fig. 6 for $L$ quartz, respectively. Some points show a deviation from the fitted curves in both figures and these are attributed to multiple scattering. The phase shift which is observed in the energy dependent azimuth scans results from the mixing parameter $\eta$ between the parity-even and parity-odd resonant events. The parity-odd resonant event is associated with the space-group \#152 and \#154 both of which have no inversion symmetry ${ }^{8}$ and is derived from the parity-odd multipole due to the strong covalent bonding between oxygen atoms with aluminum ions in berlinite, or with silicon ions in quartz.

Figure 3 shows that reflection 001 of $L$ quartz has some amount of intensity in a range from $E=1850$ to $1860 \mathrm{eV}$ comparing with the maximum at $E=1847.9 \mathrm{eV}$ while that of $L$ berlinite has rather smaller intensity in a range from $E$ $=1570$ to $1580 \mathrm{eV}$ comparing with the maximum at $E$ $=1566.8 \mathrm{eV}$. In the other words, the effect of parity-odd resonant events is more prominent for $L$ quartz than $L$ berlinite. N. Thong and D. Schwarzenbach have calculated the electron distribution around $\mathrm{Al}$ and $\mathrm{P}$ in berlinite ${ }^{19}$ using the $\mathrm{X}$-ray diffraction data and have concluded that the Al-O bond is less covalent compared to the P-O bond. This may be one of the reasons why only the $E 1 E 1$ resonant event explains the behavior of azimuth scans at the main resonant energy $E=1566.8 \mathrm{eV}$, where the intensity of the diffracted beam is the maximum. This speculation raises the possibility that a resonant diffraction of $L$ berlinite at the Phosphorus $1 s$ absorption edge $(\sim 2145 \mathrm{eV})$ would give results similar to those of $L$ quartz in terms of a phase shift at the main resonance energy. We hope the present results motivate further experiments on the role of circularly polarized resonant $\mathrm{X}$-ray diffraction for the study of chirality.

\section{SUMMARY}

We have performed resonant $\mathrm{x}$-ray diffraction on a quartz $\left(\mathrm{SiO}_{2}\right)$ and a berlinite $\left(\mathrm{AlPO}_{4}\right)$ using circularly polarized $\mathrm{X}$-rays. Space-group forbidden reflection 001 for quartz and reflection 001 and 002 for berlinite were observed in the vicinity of $\mathrm{Si} 1 s$ and $\mathrm{Al} 1 s$, respectively. The intensity of these reflections were measured as a function of the energy of incident beam, the helicity of incident beam and the azimuth angle.

We find that azimuth angle scans of reflections of the berlinite (quartz) at the $\mathrm{Al} 1 s(\mathrm{Si} 1 s)$ absorption edge where 
the intensity is at the maximum clearly show strong dependence on the helicity of the circularly polarized incident beam. The property of the azimuth scans is mostly explained by the analysis with the $E 1 E 1$ resonant event and determines the chirality from an enantiomorphic space-group pair $P 3_{1} 21$ and $P 3_{2} 21$. Experimental results show that our crystals, berlinite, and quartz, belong to space group $P 3_{2} 21$ (\#154, lefthanded screw) unambiguously.

We have observed the optical rotatory power of our samples of berlinite and quartz independently with $\mathrm{He}-\mathrm{Ne}$ laser system (wave length $\lambda=533 \mathrm{~nm}$ ). The berlinite sample shows $\gamma=-14 \pm 1$ degrees/mm, laevorotatory power (counterclockwise rotation observing toward the laser source) and the quartz sample shows $\gamma=+18 \pm 1$ degrees $/ \mathrm{mm}$, dextrorotatory power (clockwise rotation observing toward the laser source). According to Refs. 13 and 19, both crystals belong to space group $P 3_{2} 21$. This completely coincides with our observation by resonant $\mathrm{x}$-ray diffraction.
Thus, we have developed a method for an absolute determination of crystal chirality i.e., determination of a specific enantiomer of an enantiomorphic space-group pair. The technique is generally applicable to chiral motifs that occur in biomolecules, liquid crystals, ferroelectrics and antiferroelectrics, multiferroics, etc.

\section{ACKNOWLEDGMENTS}

We thank A. M. Glazer for valuable discussions about the optical rotatory power and the crystal structure. This work was partly supported by Grants-in-Aid for Scientific Research (A) Grant No. 21244049 from JSPS. The synchrotron radiation experiments were performed at beam line 17SU in SPring-8 with the approval of RIKEN (Proposal No. 20080045 and 20090012).
${ }^{1}$ S. Nishikawa and K. Matsukawa, Proc. Imp. Acad. (Tokyo) 4, 96 (1928).

${ }^{2}$ D. Coster, K. S. Knol, and J. A. Prins, Z. Phys. 63, 345 (1930).

${ }^{3}$ J. M. Bijvoet, A. F. Peerdeman, and A. J. van Bommel, Nature (London) 168, 271 (1951).

${ }^{4}$ D. H. Templeton and L. K. Templeton, Acta Crystallogr., Sect. A: Cryst. Phys., Diffr., Theor. Gen. Crystallogr. 36, 237 (1980).

${ }^{5}$ D. H. Templeton and L. K. Templeton, Acta Crystallogr., Sect. A: Cryst. Phys., Diffr., Theor. Gen. Crystallogr. 38, 62 (1982).

${ }^{6}$ V. E. Dmitrienko, Acta Crystallogr., Sect. A: Found. Crystallogr. 39, 29 (1983).

${ }^{7}$ V. E. Dmitrienko, K. Ishida, A. Kirfel, and E. N. Ovchinnikova, Acta Crystallogr., Sect. A: Found. Crystallogr. 61, 481 (2005).

${ }^{8}$ S. W. Lovesey, E. Balcar, K. S. Knight, and J. Fernández Rodríguez, Phys. Rep. 411, 233 (2005).

${ }^{9}$ Y. Tanaka, T. Takeuchi, S. W. Lovesey, K. S. Knight, A. Chainani, Y. Takata, M. Oura, Y. Senba, H. Ohashi, and S. Shin, Phys. Rev. Lett. 100, 145502 (2008).

${ }^{10}$ S. W. Lovesey, E. Balcar, and Y. Tanaka, J. Phys.: Condens. Matter 20, 272201 (2008).

${ }^{11}$ A. M. Glazer and K. Stadnicka, Acta Crystallogr., Sect. A: Found. Crystallogr. 45, 234 (1989).

${ }^{12}$ H. D. Flack and G. Bernardinelli, Acta Crystallogr., Sect. A:
Found. Crystallogr. 55, 908 (1999).

${ }^{13}$ A. M. Glazer and K. Stadnicka, J. Appl. Crystallogr. 19, 108 (1986).

${ }^{14}$ L. D. Landau and E. M. Lifshitz, Quantum Electrodynamics, 2nd ed. (Pergamon Press, Oxford, 1982), Vol. 4.

${ }^{15}$ S. W. Lovesey and S. P. Collins, X-ray Scattering and Absorption by Magnetic Materials (Clarendon Press, Oxford, 1996).

${ }^{16}$ H. Ohashi et al., AIP Conf. Proc. 879, 523 (2007).

${ }^{17}$ K. Shirasawa A. Hiraya, T. Tanaka, and H. Kitamura, Phys. Rev. ST Accel. Beams 7, 020702 (2004).

${ }^{18}$ T. Takeuchi, A. Chainani, Y. Takata, Y. Tanaka, M. Oura, M. Tsubota, Y. Senba, H. Ohashi, T. Mochiku, K. Hirata, and S. Shin, Rev. Sci. Instrum. 80, 023905 (2009).

${ }^{19}$ N. Thong and D. Schwarzenbach, Acta Crystallogr., Sect. A: Cryst. Phys., Diffr., Theor. Gen. Crystallogr. 35, 658 (1979).

${ }^{20}$ A. Rogalev, J. Goulon, F. Wilhelm, K. A. Kozlovskaya, E. N. Ovchinnikova, L. V. Soboleva, A. F. Konstantinova, and V. E. Dmitrienko, Crystallogr. Rep. 53, 384 (2008).

${ }^{21}$ B. K. Agarwal, X-ray Spectroscopy, 2nd ed. (Springer-Verlag, New York, 1991).

${ }^{22}$ Y. Tanaka, S. P. Collins, S. W. Lovesey, M. Matsunami, T. Moriwaki, and S. Shin, J. Phys.: Condens. Matter 22, 122201 (2010). 